https://helda.helsinki.fi

Cardiorespiratory fitness and heart rate recovery predict sudden cardiac death independent of ejection fraction

Hernesniemi, Jussi A.

2020-03

Hernesniemi , J A , Sipilä, K, Tikkakoski , A, Tynkkynen, J Mishra , P, Lyytikainen , L-P , Nikus , K , Nieminen , T , Lehtimaki , T \& Kähönen, M 2020 , ' Cardiorespiratory fitness and heart rate recovery predict sudden cardiac death independent of ejection fraction ', Heart , vol. 106 , no. 6 , pp. 434-440 . https://doi.org/10.1136/heartjnl-2019-315198

http://hdl.handle.net/10138/325213

https://doi.org/10.1136/heartjnl-2019-315198

cc_by_nc

acceptedVersion

Downloaded from Helda, University of Helsinki institutional repository.

This is an electronic reprint of the original article.

This reprint may differ from the original in pagination and typographic detail.

Please cite the original version. 


\section{Cardiorespiratory fitness and heart rate recovery predict sudden cardiac death independent of ejection fraction}

Hernesniemi: Prediction of sudden cardiac death by exercise testing

Jussi Hernesniemi ${ }^{1,2,3}$, Kalle Sipilä ${ }^{4}$, Antti Tikkakoski ${ }^{4}$, Juho Tynkkynen ${ }^{1,2,5}$, Pashupati Mishra ${ }^{6}$, Leo-Pekka Lyytikäinen ${ }^{1,2,3}$, Kjell Nikus ${ }^{1,2,3}$, Tuomo Nieminen ${ }^{7}$, Terho Lehtimäki ${ }^{1,3,6}$ and Mika Kähönen ${ }^{1,2,3,4}$

${ }^{1}$ Faculty of Medicine and Health Technology, Tampere University, Tampere Finland

${ }^{2}$ Tays Heart Hospital, Tampere University Hospital, Tampere Finland

${ }^{3}$ Finnish Cardiovascular Research Center Tampere, Tampere University, Tampere, Finland.

${ }^{4}$ Department of Clinical Physiology, Tampere University Hospital, University of Tampere

${ }^{5}$ Department of Radiology, Kanta-Häme Central Hospital, Hämeenlinna, Tampere, Finland

${ }^{6}$ Department of Clinical Chemistry, Fimlab Laboratories, Tampere, Finland

${ }^{7}$ Department of Internal Medicine, Päijät-Häme Central-Hospital, Lahti, Finland

Adress of Correspondence: Jussi Hernesniemi, MD, PhD, Tampere University, Tampere, Finland Address: Arvo-Building (2nd floor), Arvo Ylpön katu 34, 33520 Tampere, Finland e-mail: Jussi.hernesniemi@tuni.fi

Tel: +331164254

Total word count: 3029 


\begin{abstract}
Objective - To evaluate whether cardiorespiratory fitness (CRF) and heart rate recovery (HRR) associate with the risk of sudden cardiac death (SCD) independently of left ventricular ejection fraction (LVEF).
\end{abstract}

Methods - The Finnish Cardiovascular Study (FINCAVAS) is a prospective clinical study of patients referred to clinical exercise testing in 2001-2008 and follow-up until December 2013. Patients without pacemakers undergoing first maximal or submaximal exercise testing with cycle ergometer were included ( $\mathrm{n}=3776)$. CRF in metabolic equivalents (METs) was estimated by achieved maximal work level. HRR was defined as the reduction in heart rate one minute after maximal exertion. Adjudication of SCD was based on death certificates. LVEF was measured for clinical indications in $71.4 \%$ of the patients $(n=2697)$.

Results - Population mean age was 55.7 years (SD 13.1)(61\% men). 98 SCDs were recorded during a median follow-up of 9.1 years (6.9-10.7). Mean CRF and HRR were 7.7(SD 2.9) METs and 25(SD 12) beats/min/min. Both CRF and HRR associated with the risk of SCD in the entire study population $\left(\mathrm{HR}_{\mathrm{CRF}} 0.47[0.37-0.59], \mathrm{p}<0.001\right.$ and $\mathrm{HR}_{\mathrm{HRR}} 0.57[0.48-0.67]$, $\mathrm{p}<0.001$ with HRestimates corresponding to one SD increase in the exposure variables) and with CRF, HRR and LVEF in the same model $\left(\mathrm{HR}_{\mathrm{CRF}} 0.60[0.45-0.79], \mathrm{p}<0.001, \mathrm{HR}_{\mathrm{HRR}} 0.65[0.51-0.82], \mathrm{p}<0.001\right)$ or adjusting additionally for all significant risk factors for SCD (LVEF, sex, creatinine level, history of myocardial infarction and atrial fibrillation, corrected QT interval) (HR $\mathrm{CRF}_{0.69}$ [0.52-0.93], $\left.\mathrm{p}<0.01, \mathrm{HR}_{\mathrm{HRR}} 0.74[0.58-0.95], \mathrm{p}=0.02\right)$.

Conclusions - CRF and HRR are significantly associated with the risk of SCD regardless of LVEF. 


\section{What is already known about this subject?}

Cardiorespiratory fitness and heart rate reduction measured in clinical exercise testing have been associated with the risk of sudden cardiac death among populations undergoing clinical exercise. Earlier studies have been performed largely before the era of measuring left ventricular ejection fraction routinely in clinical work and before the use of most medical therapies aimed to reduce the risk of sudden cardiac death.

\section{What does this study add?}

The novel observations of the present contemporary study population demonstrate that the associations between cardiorespiratory fitness and heart rate reduction and sudden cardiac death are independent of left ventricular ejection fraction, which is currently the cornerstone in evaluating the risk of sudden cardiac death.

\section{How might this impact on clinical practice?}

The risk of sudden cardiac death can be evaluated by measuring cardiorespiratory fitness and heart rated reduction during clinical exercise testing despite the fact that left ventricular ejection fraction is known. They can also be used to evaluate the risk of sudden cardiac death among patients with only slightly reduced or normal ejection fraction. 


\section{INTRODUCTION}

The vast majority of all sudden cardiac deaths (SCDs) occur among individuals with no apparent heart disease or with only slightly reduced left ventricular ejection fraction (LVEF). Currently only patients with a reduced left ventricular ejection fraction $(\mathrm{LVEF}<35 \%)$ or with very rare conditions are eligible for an implantable cardioverter-defibrillator (ICD) for primary prevention of SCD given their high risk for the event [1-3].

Clinical exercise testing can be used to assess probability of coronary artery disease (CAD) and to improve prognostic evaluation [4-12]. It provides an easy way to quantify cardiorespiratory fitness (CRF) in metabolic equivalents (METs) indirectly by measuring achieved work level. Only few previous studies that have linked CRF with the risk of SCD[4,13,14]. In addition to CRF, heart rate recovery (HRR), which is very simple to assess as the difference in heart rate from the peak exercise to one minute post-exercise, has also been linked with SCD among men [4].

Currently there is no evidence of the prognostic value of exercise testing for SCD, if left ventricular ejection fraction (LVEF), which is the most significant risk factor for SCD, is included as a prognostic factor. Furthermore, previous studies $[4,13,14]$ have been performed before the era of wide spread use of statins, angiotensin-converting enzyme (ACE) inhibitors and angiotensin II receptor blockers (ARBs) in the medical management of CAD and heart failure and the use of ICDs in primary prevention of SCD [3].

The purpose of this study was to evaluate the clinical utility of CRF and HRR, both associated with the function of cardiac autonomic nervous system $[15,16]$, in predicting SCD regardless of LVEF and in a contemporary setting. For this purpose, we used data from a prospective The Finnish Cardiovascular Study (FINCAVAS) of 3776 patients undergoing clinical exercise testing between 2001 and 2008 with almost a decade of follow-up. 


\section{MATERIAL AND METHODS}

\section{Study population}

The patient population of FINCAVAS comprises all willing consecutive patients between October 2001 and December 2008 who were referred for an exercise test at Tampere University Hospital. Some patients performed multiple exercise tests but only the first technically successful exercise test for each patient was included into the study $(n=4068)$. A technically successful exercise test was defined as a test with reliably measured heart rate, blood pressure and ECG. Only patients without pacemakers or ICDs performing the exercise test on a cycle ergometer and reaching maximal or almost maximal level of exercise were included $(n=3776)$. Indications for the exercise are presented in supplementary material. The Ethics Committee of Tampere University Hospital District approved the study protocol, and all patients gave written informed consent. This study adheres to the Declaration of Helsinki. Details of the study have been published earlier in more detail [17].

\section{Patient and public involvement}

Patients were not involved in the study design or interpretation or dissemination of the results.

\section{Study protocol}

Relevant medical history was documented before the exercise. Resting ECG was recorded using Mason-Likar 12-lead prior to exercise after 10 minutes in supine position. All patients aimed to reach maximal heart rate, except patients who were evaluated soon after MI (7.6\%) where an upper heart rate limit of 120-130 beats/min was applied. The initial workload was 20 to $30 \mathrm{~W}$. The workload was increased stepwise by 10-30W every minute based on individual patient characteristics. ECGs were digitally recorded at $500 \mathrm{~Hz}$ with the CardioSoft exercise system 
(Version 4.14, GE Healthcare, Freiburg, Germany). Blood pressure was measured manually with a brachial cuff before the test, during exercise and in the recovery phase. After termination of the test, the subjects remained at the cycle ergometer seated for at least four minutes and paddled slowly against 10W resistance for a minute to allow for safe hemodynamic recovery.

\section{Measuring CRF and HRR}

CRF or exercise capacity in METs was estimated by achieved workload in reference to patients body weight using a standard formula of the GE Cardiosoft Software: METs $=(12 \times$ Workload + 3.5×bodyweight) / $3.5 \times$ bodyweight, with 1 MET corresponding to an estimated $3.5 \mathrm{ml}$ oxygen uptake/kg/min. HRR was defined as the difference between maximum heart rate during exercise and heart rate after the first minute following cessation of exercise.

\section{Measurement of LVEF}

LVEF information was available in $n=2697$ (71.4\%) study patients. LVEF values were measured for clinical indications by echocardiography, invasive contrast left ventriculography or by gated myocardial perfusion scintigraphy and the information was extracted retrospectively from patient records. If the patients had multiple measurements, the most recent measurement before the exercise test was preferred. Median time between the exercise test and LVEF measurement was 8 weeks (interquartile range [IQR] 3-56 weeks). LVEF measured after the exercise test was used only if it preceded death by at least one year and there were no measurements before the test was available.

\section{Follow-up and end-points}

The primary endpoint of this study was SCD. For the definition of SCD, death certificates listing the circumstances of the deaths and specific causes of death (based on the 10th revision of the 
International Classification of Diseases) were received from the Causes of Death Register maintained by Statistics Finland. The autopsy rate was $46 \%$ for all deaths. The adjudication committee for the definition of cause of death comprised three physicians who all reviewed the death certificates blinded to the exercise test results. SCD was defined as sudden and unexpected death by a primary cardiac cause (without any evidence or other probable cause) and occurring within approximately one hour from the onset of symptoms or if the death was not witnessed if the person had last been seen symptomless within 24 hours of the death. In $84 \%$ of the SCDs (82/98), the foremost contributing cause of death was CAD. Loss to follow-up for the main end-point data was $0 \%$.

In order to capture all possible occurrences of potentially fatal arrhythmias during the follow-up, we also formed a secondary end-point named as cardiac arrest (CA) by combining all aborted cardiac arrests with SCDs. Cardiac arrests were first screened by searching for ICD-10 based diagnoses of resuscitations and ventricular fibrillation from the hospital districts discharge diagnose database. Ventricular tachycardia without resuscitation was not considered as a cardiac arrest. Implantation of ICD devices were monitored also from hospital electronic health registry.

\section{Statistical analysis}

Main exposure variables were CRF and HRR. The prognostic significance of main exposure variables was primarily tested with a linear Cox cause-specific hazard model with other causes of death during the follow-up censored (i.e. coded as negative cases) and with time-on-study as time scale. CRF, HRR and LVEF were standardized and centered to mean (value \pm 1 depicting one standard deviation [SD] change from zero and value 0 depicting population mean) to facilitate interpretation of the results. For this reason, all hazard ratio (HR) estimates presented correspond to the change in risk related to one SD change in the exposure variable. The main exposure variables and covariates fulfilled the proportionality assumption based on correlations on survival rankings 
with Schoenfeld residuals. All factors depicting comorbidities and patient characteristics associating significantly with SCD in univariate models were considered in the multivariable analysis.

Significant risk factors were selected to the final multivariable model by a backward elimination algorithm (stepwise probability for entry 0.05 and probability of removal 0.1 ) considering preliminary all risk factor associating significantly with the risk of SCD in univariate analyses. The linearity of the associations for continuous main exposure variables were assessed by Cox regression modeling with penalized splines. Missing values for continuous variables missing less than $5 \%$ of values were replaced by multiple imputation by chained equations (missing data for HRR $n=138$ [3.7\%], serum creatinine $n=128$ [3.4\%], blood pressure values $n=5$ [0.01\%], QT and QTc interval $n=173$ [4.6\%], QRS interval $n=121$ [3.2\%]) but the main analyses were also repeated without using any imputed data and presented in the online supplement. All statistical tests were two-tailed and used an alpha level of 0.05 . Statistical analyses were performed with the R studio version 1.1383(R Foundation for Statistical Computing, Vienna, Austria) (packages: 'mice', 'ggplot2', 'survival', 'psplines'). 


\section{RESULTS}

\section{Characteristic of the study population}

Median follow-up time of the study was 9.1 years (IQR 6.9-10.7). During the follow-up 534 patients died (14.1\%) with 98 deaths classifiable as SCDs (18.4\%) and 436 deaths due to other causes $(81.6 \%)$. The differences in baseline demographics between patients who suffered a SCD, patients died of other causes and patients live at the end of the follow-up are presented in Supplementary Table 1. Additionally, during the follow-up 47 patients suffered aborted cardiac arrest and were successfully resuscitated. Seven of these patients ultimately died of SCD totaling overall 138 CAs during the follow-up period. Two percent $(n=75)$ of the population received an ICD device during the follow-up. Only two of these patients suffered SCD.

Information of LVEF was available in $71.4 \%$ of the cases $(n=2696)$. As expected, these patients were older and with more comorbidities when compared to patients with no clinical indication for LVEF measurement (Table 1.). The distributions of CRF and HRR in the study population are presented in Figure 1. 
Table 1. Population characteristics of 3776 patients of the FINCAVAS Study

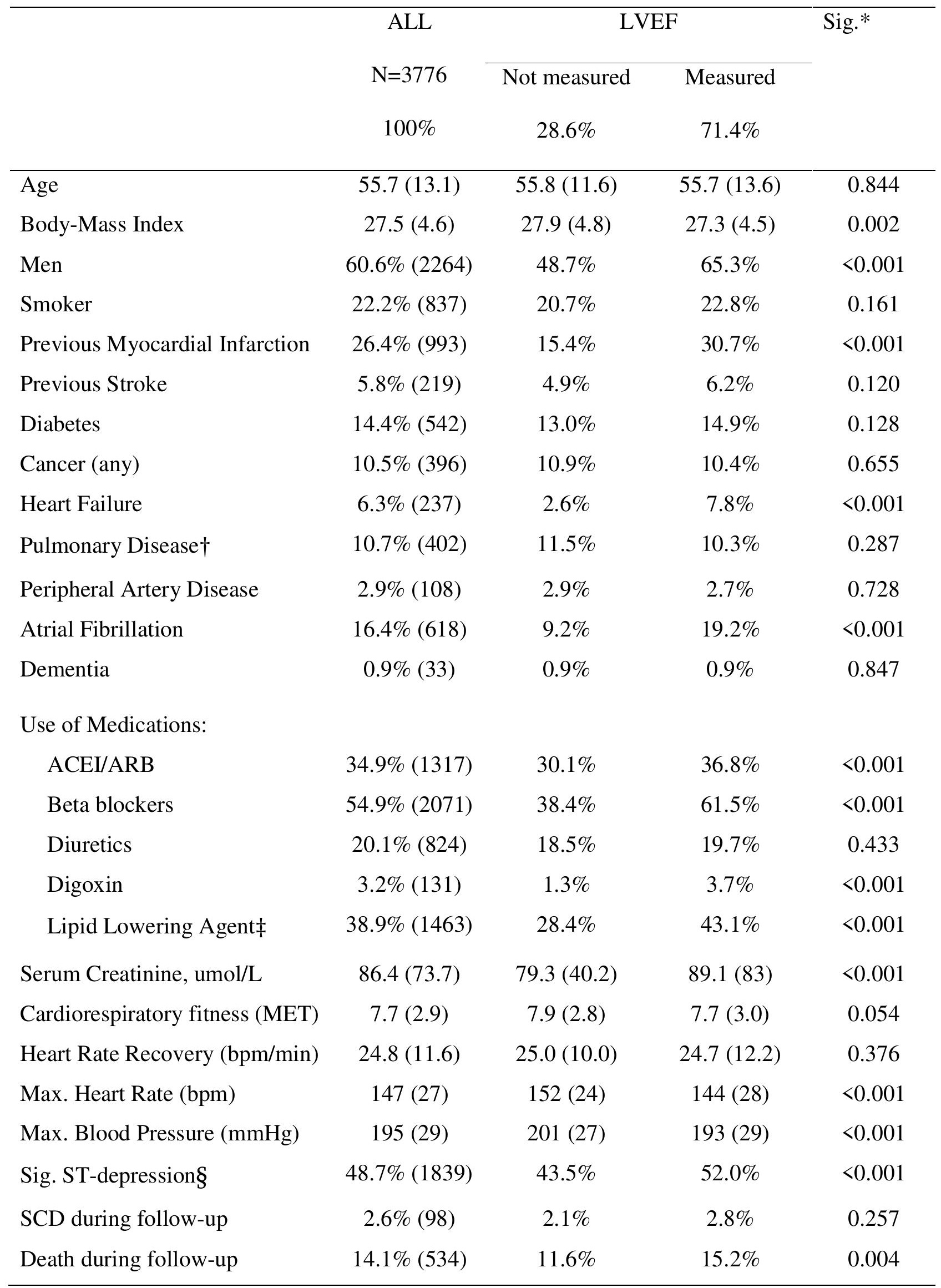


*For the comparison of patients with or without measured left ventricular ejection fraction, $\dagger$ (COPD or Asthma), $\$ 99 \%$ statin users. $\mathcal{S}$ A ST segment depression of $0.1 \mathrm{mV}$ or more during the maximal exertion or in recovery phase. Abbreviations: Sig; Significance, SCD, Sudden Cardiac Death; ACEI, Angiotensin-converting enzyme Inhibitor; ARB, Angiotensin II Receptor Blocker; LVEF, Left Ventricular Ejection Fraction; METs, Metabolic Equivalents; bpm, Beats per minute

\section{The association between CRF, HRR and SCD}

Before adjusting for any other risk factors both CRF and HRR associated with the risk of SCD in the entire study population (univariate analysis: $\mathrm{HR}_{\mathrm{CRF}} 0.47,95 \% \mathrm{CI} 0.37-0.59, \mathrm{p}=1.7 \mathrm{e}^{-10}$ and $\mathrm{HR}_{\mathrm{HRR}} 0.57,95 \%$ CI $\left.0.48-0.67, \mathrm{p}=2.5 \mathrm{e}^{-11}\right)$. After adjusting for $\mathrm{LVEF}$, both variables still remained significantly associated with SCD ( $\mathrm{HR}_{\mathrm{CRF}} 0.52,95 \%$ CI $0.39-0.68, \mathrm{p}=3.4 \mathrm{e}^{-6}$ and $\mathrm{HR}_{\mathrm{HRR}} 0.60,95 \%$ CI $\left.0.49-0.73, \mathrm{p}=3.3 \mathrm{e}^{-7}\right)$. When all three factors (CRF, HRR and LVEF) were included in the same model, all associations were significant $\left(\mathrm{HR}_{\mathrm{CRF}} 0.60,95 \% \mathrm{CI} 0.45-0.79, \mathrm{p}=2.2 \mathrm{e}^{-4}, \mathrm{HR}_{\mathrm{HRR}} 0.65,95 \%\right.$ CI $0.51-0.82, \mathrm{p}=2.9 \mathrm{e}^{-4}$ and $\mathrm{HR}_{\mathrm{LVEF}} 0.67,95 \%$ CI $\left.0.54-0.83, \mathrm{p}=2.5 \mathrm{e}^{-4}\right)$. The results remained similar when analysis was restricted to only patients with recent LVEF measurement (within three months, $\mathrm{n}=1451)\left(\mathrm{LVEF}\right.$ adjusted: $\mathrm{HR}_{\mathrm{CRF}} 0.55,95 \%$ CI 0.37-0.81, $\mathrm{p}=0.003$ and $\mathrm{HR}_{\mathrm{HRR}} 0.57,95 \%$ CI 0.39 0.82, $\mathrm{p}=0.002$ ) or restricting the analyses to subjects with no imputed data (Supplementary Table 2) Results of more extensive multivariable models are presented in Table 2., with additional adjustments made for all significant risk factors associating with SCD in the entire study population (including markers of electrophysiological instability such as QRS duration or corrected QT interval). Further adjustment for prescribed medications, did not change the results significantly despite the fact that they too are associated with CRF and HRR (Supplementary Table 3). The development of the SCD risk related to CRF and HRR in the entire study population is demonstrated in Figure 2., showing a tendency for non-linear association. The trends were similar 
after adjusting for LVEF (Supplementary Figure 1). The associations between main exposure variables and other risk factors with the risk of dying to any other cause are presented in Supplementary Table 4 and the combined effects of CRF and HRR on the risk of SCD are presented in Supplementary Table 5. 
Table 2. The association between baseline risk factors (comorbidities and patient characteristics) and sudden cardiac death in the FINCAVAS study population ( $n=3776)$. Information of left ventricular ejection fraction (LVEF) was available in $71.4 \%(n=2697)$ of the study population.

Hazard ratio (95\% Confidence Interval)*

\begin{tabular}{|c|c|c|c|}
\hline & Univariate & Multivariable & Multivariable with LVEF \\
\hline Age & $1.03(1.01-1.05)$ & - & - \\
\hline Body-Mass Index & $1.08(1.04-1.12)$ & - & - \\
\hline Sex (Male) & $2.90(1.74-4.83)$ & $2.09(1.23-3.55)$ & $2.09(1.11-3.95)$ \\
\hline Smoking & $1.26(0.81-1.96)$ & - & - \\
\hline Previous MI & $5.09(3.36-7.69)$ & $3.72(2.41-5.73)$ & $3.80(2.28-6.34)$ \\
\hline Previous Stroke & $1.95(1.01-3.75)$ & - & - \\
\hline Diabetes & $2.61(1.69-4.03)$ & - & - \\
\hline Cancer (any) & $1.59(0.89-2.85)$ & - & - \\
\hline Heart Failure & $4.32(2.61-7.13)$ & - & - \\
\hline Pulmonary Disease $\dagger$ & $2.20(1.34-3.59)$ & $1.80(1.09-2.96)$ & $1.76(0.99-3.13)$ \\
\hline Peripheral Artery Disease & $3.58(1.73-7.37)$ & & - \\
\hline Atrial Fibrillation & $2.48(1.62-3.80)$ & $1.93(1.23-3.02)$ & $1.86(1.15-3.18)$ \\
\hline Dementia due to any cause & $1.28(0.18-9.17)$ & - & - \\
\hline Serum Creatinine & $1.15(1.06-1.25)$ & $1.14(1.03-1.27)$ & $1.12(1.01-1.25)$ \\
\hline Cardiorespiratory fitness & $0.47(0.37-0.59)$ & $0.63(0.48-0.81)$ & $0.69(0.52-0.93)$ \\
\hline Heart Rate Recovery & $0.57(0.49-0.68)$ & $0.74(0.60-0.92)$ & $0.74(0.58-0.95)$ \\
\hline Max. Heart Rate & $0.57(0.49-0.68)$ & - & - \\
\hline Max. Blood Pressure & $0.61(0.50-0.75)$ & - & - \\
\hline Sig. ST-depression $\ddagger$ & $0.60(0.40-0.91)$ & - & - \\
\hline QTc-interval & $1.37(1.15-1.65)$ & - & $1.25(1.01-1.51)$ \\
\hline QRS duration & $1.37(1.21-1.57)$ & $1.25(1.07-1.45)$ & - \\
\hline LVEF & $0.61(0.49-0.76)$ & NA & - \\
\hline $\mathrm{LVEF} \leq 35 \% \S$ & $6.41(3.58-11.48)$ & NA & $3.35(1.84-6.10)$ \\
\hline
\end{tabular}


*Hazard ratio estimates correspond to a one standard deviation increase in exposure variables for continuous risk factors except for age for which the hazard estimate corresponds to a one-year increase. Risk estimates for multivariable analyses are reported only for variables associating with SCD with a p-value of $<0.05$ in the final model. Significant risk factors were selected to the model by a backward elimination algorithm. †Chronic Obstructive Pulmonary Disease or Asthma. $¥$ At least 0.1mV ST-segment depression during or after the exercise. $\$$ Multivariable model without LVEF as continuous variable in the model

\section{Interactions and sensitivity analyses}

The unadjusted associations between main exposure variables and SCD were all consistent and significant among different patient subpopulations stratified by the use of medications and with different indications for the exercise test (Table 3) and among patients with LVEF $>35 \%$. Interestingly, interaction analyses revealed that poor HRR might be a stronger risk factor among women when compared to men ( $\mathrm{p}=0.01$ for interaction) and also when HRR was measured from an almost maximal exercise test when compared to maximal exercise test ( $\mathrm{p}=0.02$ for interaction)(Table 3). 
Table 3. The unadjusted association between exercise test parameters and sudden cardiac death among different subgroups of patients undergoing clinical exercise testing. Results presented in bold text have nominally significant $(p<0.05)$ interaction suggesting significantly different hazard ratios between subgroups.

Hazard Ratios (95\% Confidence Interval)

Cardiorespiratory Fitness Heart Rate Recovery

All, $n=3776$

$0.47(0.37-0.59)$

$0.57(0.49-0.68)$

Sex

Men, $n=2262$

$0.43(0.33-0.55)$

$0.65(0.55-0.79)$

Women, $\mathrm{n}=1470$

$0.46(0.25-0.84)$

$0.32(0.19-0.55)$

LVEF function
LVEF $>35 \%, \mathrm{n}=2584 * *$
$0.55(0.41-0.73)$
$0.62(0.51-0.75)$
LVEF $<35 \%, \mathrm{n}=113$
$0.34(0.15-0.81)$
$0.76(0.40-1.44)$

Users of different medications:

Beta-blockers, $n=2071$

$0.42(0.31-0.57)$

$0.55(0.44-0.70)$

ACEI or ARB, $n=1317$

$0.51(0.37-0.70)$

$0.55(0.42-0.74)$

Lipid lowering agent*, $n=1463$

$0.52(0.36-0.75)$

$0.58(0.43-0.77)$

Patients with different indications:

Suspicion of CAD, $n=1603$

$0.52(0.33-0.79)$

$0.42(0.27-0.63)$

Evaluation of drug therapy, $n=459$

$0.53(0.31-0.89)$

$0.53(0.32-0.82)$

Evaluation prior to an operation, $n=316$

$0.52(0.23-1.18)$

$0.54(0.26-1.12)$

Evaluation of arrhythmia, $n=869$

$0.41(0.25-0.65)$

$0.59(0.45-0.77)$

Evaluation after $\mathrm{MI}, \mathrm{n}=256$

$0.29(0.13-0.65)$

$0.53(0.27-1.05)$

Evaluation of work capacity, $n=897$

$0.41(0.17-0.99)$

$0.68(0.47-0.98)$

Patients reaching different level of exertion**

Maximal, $\mathrm{n}=3040$

$0.49(0.38-0.64)$

$0.64(0.53-0.78)$

Almost Maximal, $\mathrm{n}=736$

$0.43(0.26-0.71)$

$0.36(0.23-0.58)$

*99\% statins, $* *$ Assessed by the supervising physician after termination of the test. 


\section{Association between CRF and HRR and CA}

Similarly, both CRF and HRR associated with the risk of CA in univariate analyses and after adjusting for LVEF (univariate analysis: $\mathrm{HR}_{\mathrm{CRF}} 0.54,95 \% \mathrm{CI} 0.45-0.66, \mathrm{p}=3.3 \mathrm{e}^{-10}$ and $\mathrm{HR}_{\mathrm{HRR}} 0.66$, 95\% CI 0.57-0.78, $\mathrm{p}=2.9 \mathrm{e}^{-7}$ and LVEF adjusted HR $\mathrm{CRF} 0.61,95 \%$ CI $0.49-0.76, \mathrm{p}=1.3 \mathrm{e}^{-5}$ and $\mathrm{HR}_{\mathrm{HRR}}$ $0.71,95 \%$ CI $\left.0.59-0.86, p=2.7 \mathrm{e}^{-4}\right)$. All three factors persisted as significant also in the same model $\left(\mathrm{HR}_{\mathrm{CRF}} 0.66,95 \%\right.$ CI $0.52-0.82, \mathrm{p}=2.8 \mathrm{e}^{-4}, \mathrm{HR}_{\mathrm{HRR}} 0.78,95 \%$ CI $0.64-0.95, \mathrm{p}=0.013$ and $\mathrm{HR}_{\mathrm{LVEF}}$ $0.62,95 \%$ CI $\left.0.52-0.74, \mathrm{p}=1.3 \mathrm{e}^{-7}\right)$.

\section{DISCUSSION}

The results of this large prospective follow-up study of patients undergoing clinical exercise testing show that CRF and HRR are significant predictors of SCD regardless of LVEF in a contemporary patient population. Furthermore, both CRF and HRR associate with the risk of SCD among patients with normal or slightly reduced LVEF $(>35 \%)$.

Our results are in line with previous findings from other cohorts $[13,14]$. In the Kuopio Ischemic Heart Disease study of 2368 randomly selected middle aged Finnish men undergoing cycle ergometer testing in 1980s, one MET increase in CRF associated with an adjusted $\sim 22 \%$ relative decrease in SCD (17 years of follow-up). In the Aerobics Center Longitudinal Study of self referred subjects $(n=55,456)$ undergoing treadmill testing between the years 1974 and 2002, the corresponding number was $\sim 14 \%$ [14]. Our results are in line with an observed adjusted $\sim 17 \%$ lower risk of SCD for one MET increase (extrapolated from the linear risk estimate). We also replicate the seminal results of Jouven et al. showing that among 5713 men undergoing exercise 
testing between 1967 and 1972, reduced HRR predicted sudden death due to MI during a 23-year follow-up [4].

Adding to the findings of previous studies, we observed that the association between CRF and HRR with SCD is also independent of LVEF, which is currently the cornerstone in guiding ICD-therapy for the primary prevention of SCD [1,3]. Furthermore, the association is significant in a population with LVEF $>35 \%$. These patients are not generally eligible for ICD implantation for primary prevention of SCD despite the fact that the majority of SCDs occur among similar individuals of the general the population [1]. Similarly, in our study population approximately $80 \%$ of all SCDs occurred among subjects with LVEF $>35 \%$. Previously, the combination of reduced CRF and HRR has been shown to predict cardiovascular death and hospitalization due to congestive heart failure despite LVEF [18]. HRR has been also shown to associate with mortality independently of angiographic severity of CAD which is the underlying condition in most SCD cases and a significant contributor to LVEF reduction in the post-MI setting[19]. Similarly, HRR has been associated directly with mortality regardless of LVEF measured by stress echocardiography [20].

According to previous studies, the relative risk of SCD seems to be higher when approaching lower levels of CRF or HRR (i.e. possible deviation from linearity)[4,13,14]. These trends seem very similar to the risk functions previously observed in regards to overall and cardiovascular mortality $[12,20]$ and are also in line with our results. We also observed that the risk associated with poor HRR might be even stronger when measured after the exercise test is terminated before reaching absolute maximal exertion. This finding indicating a higher risk associated with poor HRR if present already in submaximal exertion is plausible but may also be a false positive given the number of interactions tested. Similarly, the association between HRR and SCD seemed stronger among women, but given the low number of SCDs among women $(n=17)$ the 
observation should be interpreted with caution. There are no prior studies that could validate this observation.

The exact mechanisms by which CRF and HRR affect the risk of SCD are beyond the scope of this epidemiological study. However, previous evidence suggests that aside corresponding with favorable overall cardiovascular risk profile, good CRF is associated with a better cardiac autonomic function, which in turn may reduce the risk of fatal ventricular arrhythmias $[13,16]$. Similarly, HRR is considered a good indicator of parasympathetic function [15] even regardless of angiographic verified coronary artery status[19]. Considering this, it is plausible that, in the event of possible predisposing event such as an ischemic attack, both good CRF and HRR can provide protection from fatal arrhythmias.

The other novelty of our study is the replication of the main findings $[4,13,14]$ in a contemporary patient population subjected to most medical therapies currently available for reducing the risk of SCD (including beta-blockers, ACE inhibitors or ARBs and by statins)[3]. Previous studies have been conducted among patients treated almost entirely before the era of widespread use of these medications with populations undergoing exercise tests from the end of 1960 s to the end of the last millennia $[4,13,14]$.

CRF and HRR are also strong predictors of overall mortality [5,12,21]. This means that the prognostic utility of CRF and HRR might be compromised when considering interventions targeted specifically to reduce overall mortality by preventing SCD given the high risk of competing events that are not modifiable by any specific therapies aimed to reduce the risk of SCD. For this reason, other factors should also be considered in overall risk assessment. In the present study population, age, dementia due to any neurodegenerative disease and cancer all associated with the risk of dying due to other causes but not with the risk of SCD. It is plausible that CRF and HRR might be useful in guiding primary prevention of SCD among patients who have good functional status but not significantly burdened by other comorbidities. 


\section{Limitations}

The foremost limitation of the present study is that instead of measuring LVEF systematically at the time of the exercise tests, the LVEF information was measured only in patients with a clear clinical indication (71.4\% of the population) and the information was extracted from patient records retrospectively. Despite the fact that patients with or without measured LVEF had substantially different risk profiles, associations between SCD and CRF and HRR seemed similar in both groups.

The strengths of the present study include a large sample size resulting in reliable and consistent results as well as excellent coverage for the follow-up (no drop-outs). Due to Finnish legislature and centralized registry for recording causes of death we were able to determine the mode of death in all cases (sudden or not). For all patients who died, a full disclosure review of the death certificate was performed and in all cases with un-witnessed death or if the death was otherwise unexpected despite underlying conditions, the cause of death was determined by a medicolegal autopsy. The results were also confirmed using a secondary endpoint of all cardiac arrests (SCD combined with cardiac arrests leading to successful resuscitation).

In conclusion, among subjects undergoing clinical exercise testing, CRF and HRR are significant predictors of SCD independently of LVEF. CRF and HRR are also significant prognostic factors for SCD among patients with only slightly reduced or normal LVEF. Further prospective trials are needed to verify the actual clinical value of CRF and HRR when considering primary prevention of SCD. 


\section{ACKNOWLEDGEMENTS}

The Finnish Cardiovascular Study (FINCAVAS) has been financially supported by the Competitive Research Funding of the Tampere University Hospital (Grant 9M048 and 9N035), the Finnish Cultural Foundation, the Finnish Foundation for Cardiovascular Research, the Emil Aaltonen Foundation, Finland, the Tampere Tuberculosis Foundation, and EU Horizon 2020 (grant 755320 for TAXINOMISIS).

\section{DISCLOSURES}

The authors declare no competing interests.

\section{AUTHOR STATEMENT}

The Corresponding Author has the right to grant on behalf of all authors and does grant on behalf of all authors, an exclusive licence (or non exclusive for government employees) on a worldwide basis to the BMJ Publishing Group Ltd and its Licensees to permit this article (if accepted) to be published in HEART editions and any other BMJPGL products to exploit all subsidiary rights 


\section{References}

1 Wellens HJJ, Schwartz PJ, Lindemans FW, et al. Risk stratification for sudden cardiac death: Current status and challenges for the future. Eur Heart J Published Online First: 2014. doi:10.1093/eurheartj/ehu176

2 Priori SG, Blomström-Lundqvist C, Mazzanti A. 2015 ESC Guidelines for the management of patients with ventricular arrhythmias and the prevention of sudden cardiac death. Eur Heart J Published Online First: 2015. doi:10.1093/europace/eul108

3 Al-Khatib SM, Stevenson WG, Ackerman MJ, et al. 2017 AHA/ACC/HRS Guideline for Management of Patients With Ventricular Arrhythmias and the Prevention of Sudden Cardiac Death: Executive Summary. Circulation 2018;138:e210-71. doi:10.1161/CIR.0000000000000548

4 Jouven X, Empana J-P, Schwartz PJ, et al. Heart-Rate Profile during Exercise as a Predictor of Sudden Death. N Engl J Med 2005;352:1951-8. doi:10.1056/NEJMoa043012

5 Cole CR, Blackstone EH, Pashkow FJ, et al. Heart Rate Recovery Immediately After Exercise as a Predictor of Mortality. N Engl J Med 1999;28:1351-7. doi:10.1056/NEJM199910283411804

6 Laukkanen JA, Mäkikallio TH, Rauramaa R, et al. Asymptomatic ST-segment depression during exercise testing and the risk of sudden cardiac death in middle-aged men: A population-based follow-up study. Eur Heart J 2009;30:558-65. doi:10.1093/eurheartj/ehn584

7 Hagnas MJ, Lakka TA, Kurl S, et al. Cardiorespiratory fitness and exercise-induced ST segment depression in assessing the risk of sudden cardiac death in men. Heart 2017;103:383-9. doi:10.1136/heartjnl-2015-309217

8 Mundal R, Kjeldsen SE, Sandvik L, et al. Exercise blood pressure predicts cardiovascular 
mortality in middle-aged men. Hypertension 1994;24:56-62. doi:10.1161/01.HYP.24.1.56

9 Hedberg P, Ohrvik J, Lonnberg I, et al. Augmented blood pressure response to exercise is associated with improved long-term survival in older people. Heart 2009;95:1072-8. doi:10.1136/hrt.2008.162172

10 Naughton J, Dorn J, Oberman A, et al. Maximal exercise systolic pressure, exercise training, and mortality in myocardial infarction patients. Am J Cardiol 2000;85:416-20. doi:10.1016/S0002-9149(99)00765-1

11 Fletcher GF, Ades PA, Kligfield P, et al. Exercise standards for testing and training. Circulation 2013;128:873-934. doi:10.1161/CIR.0b013e31829b5b44

12 Ross R, Blair SN, Arena R, et al. Importance of Assessing Cardiorespiratory Fitness in Clinical Practice: A Case for Fitness as a Clinical Vital Sign: A Scientific Statement from the American Heart Association. Circulation 2016;134:e653-99. doi:10.1161/CIR.0000000000000461

13 Laukkanen JA, Mkikallio TH, Rauramaa R, et al. Cardiorespiratory fitness is related to the risk of sudden cardiac death: A population-based follow-up study. J Am Coll Cardiol 2010;56:1476-83. doi:10.1016/j.jacc.2010.05.043

14 Jiménez-Pavón D, Artero EG, Lee D, et al. Cardiorespiratory Fitness and Risk of Sudden Cardiac Death in Men and Women in the United States: A Prospective Evaluation From the Aerobics Center Longitudinal Study. Mayo Clin Proc 2016;91:849-57. doi:10.1016/j.mayocp.2016.04.025

15 Imai K, Sato H, Hori M, et al. Vagally mediated heart rate recovery after exercise is accelerated in athletes but blunted in patients with chronic heart failure. J Am Coll Cardiol 1994;24:1529-35. doi:10.1016/0735-1097(94)90150-3

16 Tulppo MP, Mäkikallio TH, Seppänen T, et al. Vagal modulation of heart rate during exercise: effects of age and physical fitness. Am J Physiol 1998;274:H424-9. 
17 Nieminen T, Lehtinen R, Viik J, et al. The Finnish Cardiovascular Study (FINCAVAS): Characterising patients with high risk of cardiovascular morbidity and mortality. BMC Cardiovasc Disord 2006;6. doi:10.1186/1471-2261-6-9

18 Kiviniemi AM, Lepojärvi S, Kenttä T V., et al. Exercise Capacity and Heart Rate Responses to Exercise as Predictors of Short-Term Outcome Among Patients With Stable Coronary Artery Disease. Am J Cardiol Published Online First: 2015. doi:10.1016/j.amjcard.2015.08.014

19 Vivekananthan DP, Blackstone EH, Pothier CE, et al. Heart rate recovery after exercise is a predictor of mortality, independent of the angiographic severity of coronary disease. $J \mathrm{Am}$ Coll Cardiol Published Online First: 2003. doi:10.1016/S0735-1097(03)00833-7

20 Watanabe J, Thamilarasan M, Blackstone EH, et al. Heart rate recovery immediately after treadmill exercise and left ventricular systolic dysfunction as predictors of mortality: the case of stress echocardiography. Circulation 2001;104:19116.http://www.ncbi.nlm.nih.gov/pubmed/11602493

21 Nishime EO, Cole CR, Blackstone EH, et al. Heart rate recovery and treadmill exercise score as predictors of mortality in patients referred for exercise ECG. J Am Med Assoc 2000;284:1392-8. doi:10.1001/jama.284.11.1392 


\section{FIGURES}
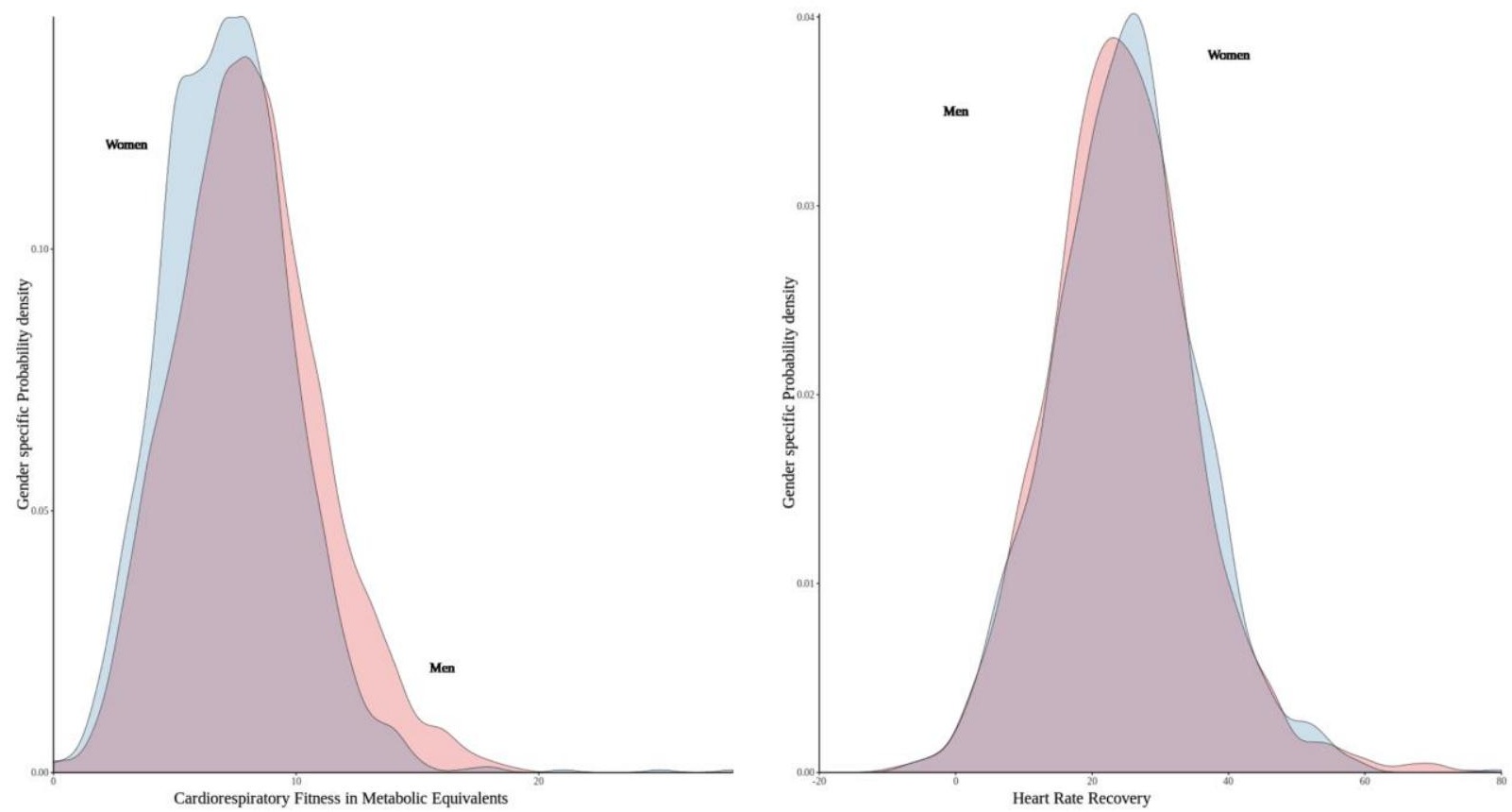

Figure 1. Cardiorespiratory fitness and heart rate recovery distributions in the FINCAVAS study population of 3776 patients undergoing exercise testing for clinical indications
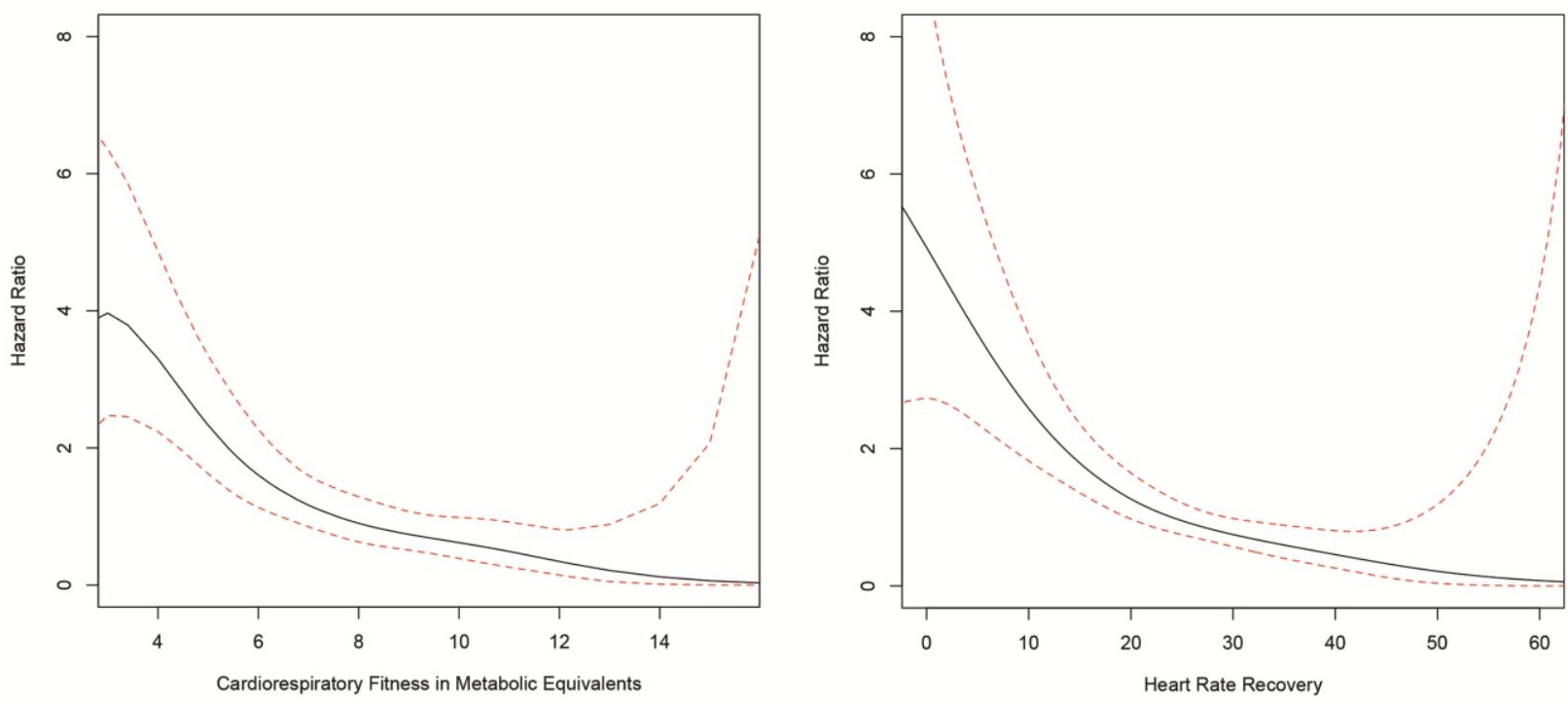

Figure 2. The association curve for the sudden cardiac death risk by cardiorespiratory fitness and heart rate recovery in the follow-up of the prospective FINCAVAS study population of 3776 patients undergoing clinical exercise testing for clinical indications. The risk level is standardized to mean values for both exposure variables (7.7 METs and 25bpm/minute). 
Supplementary Material

Cardiorespiratory fitness and heart rate recovery predict sudden cardiac death independent of left ventricular ejection fraction - The FINCAVAS Study

Hernesniemi et al.

SUPPLEMENTARY METHODS

Indications for the exercise test

Indications for the test were suspicion of CAD (43\%), evaluation of work capacity (24\%), arrhythmias (25\%), adequacy of CAD drug therapy (13\%) and evaluation prior to an invasive procedure $(9 \%)$ or after myocardial infarction (MI) $(7.6 \%)$. Some patients had more than one indication.

\section{Reasons for termination of the test and evaluation of achieved work level}

The reasons for termination of the test were chest pain (6\%), dyspnoea (12\%), fatigue (64\%), ECG changes $(1 \%)$, blood pressure changes $(1 \%)$ or other reasons $(10 \%)$. The objective exercise level as defined by the supervising physician was maximal in $81 \%$ or almost maximal in $19 \%$ of the tests. The achieved work level (classified as maximal, almost maximal, reasonable or small) was assessed by the supervising physician using patient's reported level of exhaustion by the Borg's scale and clinical judgement of the reasons for termination and perceived patient performance. 
Supplementary Table 1. The distribution of baseline demographics between patients who were alive or had died some other form of death and patients who died a sudden cardiac death during the follow-up of the FINCAVAS cohort.

\begin{tabular}{|c|c|c|c|}
\hline Status at the end of follow-up: & Alive $(n=3242)$ & $\operatorname{SCD}(n=98)$ & Other Death $(n=436)$ \\
\hline Age & $54.2(12.8)$ & $60.0(11.1)$ & $65.9(10.3)$ \\
\hline Body-Mass Index & $27.4(4.6)$ & $29.2(5.1)$ & $27.9(4.4)$ \\
\hline $\operatorname{Men}(\%)$ & $58.9 \%$ & $81.6 \%$ & $67.9 \%$ \\
\hline Smoker & $21.7 \%$ & $27.6 \%$ & $24.3 \%$ \\
\hline Previous MI & $22.6 \%$ & $64.3 \%$ & $45.6 \%$ \\
\hline Previous Stroke & $4.1 \%$ & $10.2 \%$ & $17.9 \%$ \\
\hline Diabetes & $12.3 \%$ & $29.6 \%$ & $26.4 \%$ \\
\hline Cancer (any) & $6.6 \%$ & $13.3 \%$ & $38.8 \%$ \\
\hline Heart Failure & $4.0 \%$ & $19.4 \%$ & $20.6 \%$ \\
\hline Pulmonary Disease* & $9.5 \%$ & $20.4 \%$ & $17.4 \%$ \\
\hline Peripheral Artery Disease & $1.7 \%$ & $8.2 \%$ & $10.6 \%$ \\
\hline Atrial Fibrillation & $14.8 \%$ & $31.6 \%$ & $24.3 \%$ \\
\hline Dementia & $0.1 \%$ & $1.0 \%$ & $6.7 \%$ \\
\hline \multicolumn{4}{|l|}{ Use of Medications: } \\
\hline ACEI/ARB & $32.1 \%$ & $66.3 \%$ & $48.6 \%$ \\
\hline Beta blockers & $51.9 \%$ & $77.6 \%$ & $71.6 \%$ \\
\hline Diuretics & $16.5 \%$ & $42.9 \%$ & $35.1 \%$ \\
\hline Digoxin & $2.2 \%$ & $14.3 \%$ & $6.7 \%$ \\
\hline Lipid Lowering Agent** & $37.4 \%$ & $52.0 \%$ & $46.8 \%$ \\
\hline Serum Creatinine, umol/L & $82(60)$ & $113(132)$ & $113(121)$ \\
\hline $\begin{array}{l}\text { Cardiorespiratory fitness } \\
\text { (METs) }\end{array}$ & $8.1(2.8)$ & $5.9(2.3)$ & $5.8(2.6)$ \\
\hline Heart Rate Recovery & $25.8(11.2)$ & $18.4(10.5)$ & $18.4(12.6)$ \\
\hline Max. Heart Rate (bpm) & $150(26)$ & $130(29)$ & $126(27)$ \\
\hline Max. Blood Pressure (mmHg) & $197(28)$ & $182(34)$ & $183(30)$ \\
\hline Sig. ST-depression*** & $51.2 \%$ & $37.9 \%$ & $40.5 \%$ \\
\hline $\mathrm{LVEF} \leq 35 \% * * * *$ & $3.1 \%(\mathrm{n}=70)$ & $18.7 \%(n=14)$ & $8.6 \%(n=29)$ \\
\hline
\end{tabular}

*Chronic Obstructive Pulmonary Disease or Asthma

$* * 99.4 \%$ statins and $0.6 \%$ other

***At least $0.1 \mathrm{mV}$ ST-segment depression during or after the exercise

**** Data available in $71 \%$ of the population. 
Supplementary Table 2. Replication of main results without imputed data. Missing data for HRR $\mathrm{n}=138(3.7 \%)$, serum creatinine $\mathrm{n}=128(3.4 \%)$, blood pressure values $\mathrm{n}=5(0.01 \%)$, QT and QTc interval $n=173(4.6 \%)$, QRS interval $n=121(3.2 \%)$ of the participants of the FINCAVAS study.

Hazard Ratio (95\% Confidence Interval)

\begin{tabular}{ll}
\hline HRR (univariate) $\mathrm{n}=3638$ & $0.58(0.49-0.68)$ \\
HRR (adjusted with LVEF), $\mathrm{n}=2436$ & $0.56(0.43-0.72)$ \\
HRR (adjusted with LVEF and CRF), $\mathrm{n}=2601$ & $0.64(0.51-0.81)$ \\
HRR (full model without LVEF)*, $\mathrm{n}=3521$ & $0.71(0.57-0.89)$ \\
HRR (full model)*, $\mathrm{n}=2522$ & $0.72(0.56-0.92)$
\end{tabular}

CRF (adjusted with LVEF and HRR) $n=2601$

$0.61(0.46-0.80)$

CRF (full model without LVEF)*, $n=3521$

$0.65(0.50-0.84)$

CRF (full model)*, $\mathrm{n}=2522$

$0.69(0.51-0.92)$

*As in Table 2 of the main article, model adjusted with sex, history of myocardial infarction, prevalent pulmonary disease, atrial fibrillation, serum creatinine, LVEF and CRF/HRR). Abbreviations: HRR, Heart rate reduction; LVEF, left ventricular ejection fraction; CRF, Cardiorespiratory Fitness. 
Supplementary Table 3. The association between baseline risk factors (comorbidities and patient characteristics and medications) and sudden cardiac death in the FINCAVAS study population $(\mathrm{n}=3776)$. Information of left ventricular ejection fraction (LVEF) was available in $71.4 \%(\mathrm{n}=2697)$ of the study population.

Hazard ratio (95\% Confidence Interval)

Lipid Lowering Agent

Cardiorespiratory fitness

Heart Rate Recovery

QTc-interval

QRS duration

LVEF

LVEF $\leq 35 \% \S$

\begin{tabular}{lcc}
\hline Sex (Male) & $2.11(1.23-3.64)$ & $1.90(1.01-3.56)$ \\
Previous MI & $3.64(2.28-5.81)$ & $3.43(1.99-5.94)$ \\
Pulmonary Disease $\dagger$ & $1.69(1.02-2.79)$ & $1.75(0.98-3.13)$ \\
Atrial Fibrillation & $1.64(1.02-2.63)$ & $1.67(0.97-2.86)$ \\
Serum Creatinine & $1.11(1.00-1.24)$ & $1.10(0.96-1.23)$ \\
ACEI/ARB & $1.98(1.21-3.06)$ & $2.32(1.33-4.07)$ \\
Beta blockers & $0.85(0.50-1.43)$ & $0.66(0.36-1.20)$ \\
Diuretics & $1.52(0.96-2.41)$ & $1.55(0.92-2.62)$ \\
Digoxin & $2.05(1.09-3.86)$ & $1.34(0.63-2.88)$ \\
Lipid Lowering Agent & $0.93(0.60-1.45)$ & $1.13(0.67-1.88)$ \\
Cardiorespiratory fitness & $0.70(0.53-0.91)$ & $0.74(0.55-1.00)$ \\
Heart Rate Recovery & $0.79(0.64-0.99)$ & $0.78(0.61-1.01)$ \\
QTc-interval & NA & $1.23(1.01-1.50)$ \\
QRS duration & - & - \\
LVEF & $1.19(1.02-1.41)$ & - \\
LVEF $\leq 35 \% \S$ & NA & \\
\hline
\end{tabular}

Multivariable

3.64 (2.28-5.81)

$1.69(1.02-2.79)$

$1.64(1.02-2.63)$

1.11 (1.00-1.24)

$1.98(1.21-3.06)$

$0.85(0.50-1.43)$

$1.52(0.96-2.41)$

2.05 (1.09-3.86)

$0.93(0.60-1.45)$

$0.70(0.53-0.91)$

0.79 (0.64-0.99)

Multivariable with LVEF 
Supplementary Table 4. The association between baseline demographics and mortality due to causes other than sudden cardiac death in the FINCAVAS study population. Risk estimates for only statistically significant or borderline significant $(\mathrm{p}<0.1)$ variables in the multivariable analyses are reported. Significant risk factors were selected to the model by a backward elimination algorithm.

Hazard ratio (95\% Confidence Interval)*

\begin{tabular}{lcc}
\hline & Univariate & Multivariable \\
& & \\
\hline Age & $1.09(1.08-1.90)$ & $1.05(1.03-1.06)$ \\
Body-Mass Index & $1.02(1.00-1.04)$ & - \\
Men (\%) & $1.37(1.12-1.68)$ & $1.27(1.01-1.58)$ \\
Smoker & $1.04(0.84-1.30)$ & $1.39(1.10-1.75)$ \\
Previous MI & $2.36(1.95-2.85)$ & $1.48(1.20-1.81)$ \\
Previous Stroke & $3.76(2.94-4.80)$ & $1.45(1.11-1.89)$ \\
Diabetes & $2.24(1.81-2.77)$ & - \\
Cancer (any) & $6.79(5.60-8.23)$ & $4.37(3.56-5.35)$ \\
Heart Failure & $4.82(3.81-6.08)$ & $1.96(1.52-2.54)$ \\
Pulmonary Disease** & $1.82(1.42-2.36)$ & - \\
Peripheral Artery Disease & $4.86(3.58-6.59)$ & $1.62(1.17-2.24)$ \\
Atrial Fibrillation & $1.74(1.40-2.16)$ & - \\
Dementia due to any cause & $9.03(6.19-13.16)$ & $3.42(2.29-5.12)$ \\
Serum Creatinine, umol/L & $1.15(1.11-1.20)$ & $1.17(1.11-1.23)$ \\
Cardiorespiratory fitness (METs) & $0.46(0.41-0.52)$ & $0.74(0.65-0.85)$ \\
Heart Rate Recovery & $0.58(0.53-0.62)$ & $0.84(0.75-0.94)$ \\
Max. Heart Rate (bpm) & $0.49(0.45-0.54)$ & - \\
Max. Blood Pressure (mmHg) & $0.65(0.59-0.71)$ & $0.90(0.81-1.00)$ \\
Sig. ST-depression*** & $0.66(0.55-0.80)$ & - \\
\hline
\end{tabular}

*Hazard ratio estimates correspond to a one standard deviation increase in exposure variables for continuous risk factors except for age for which the hazard estimate correspond to a one year increase.

${ }^{* *}$ Chronic Obstructive Pulmonary Disease or Asthma

***At least $0.1 \mathrm{mV}$ ST-segment depression during or after the exercise. 
Supplementary Table 5. The unadjusted hazard ratios associated with different combinations of CRF and HRR levels. The population is stratified to equal size tertiles according to CRF and HRR values and the risk associated with different number of risk points are presented. Each subject is assigned risk points by their level of risk according to CRF and HRR tertile stratification. The highest tertile for both yields no risk points whereas position in the middle tertile equals one risk point and position in the lowest risk tertile two risk points.

Hazard ratio (95\% Confidence Interval)

\begin{tabular}{lc}
\hline Reference (zero risk points) & 1.00 \\
One risk point & $1.92(0.61-6.04)$ \\
Two risk points & $4.00(1.39-11.45)$ \\
Three risk points & $4.61(1.58-13.50)$ \\
Four risk points & $10.22(3.65-28.64)$ \\
\hline
\end{tabular}


Supplemental Figure 1. The association curve for the sudden cardiac death risk by cardiorespiratory fitness and heart rate recovery after adjusting for left ventricular ejection fraction. The risk level is standardized to mean values for both exposure variables (7.7 METs and 24.7bpm/minute).
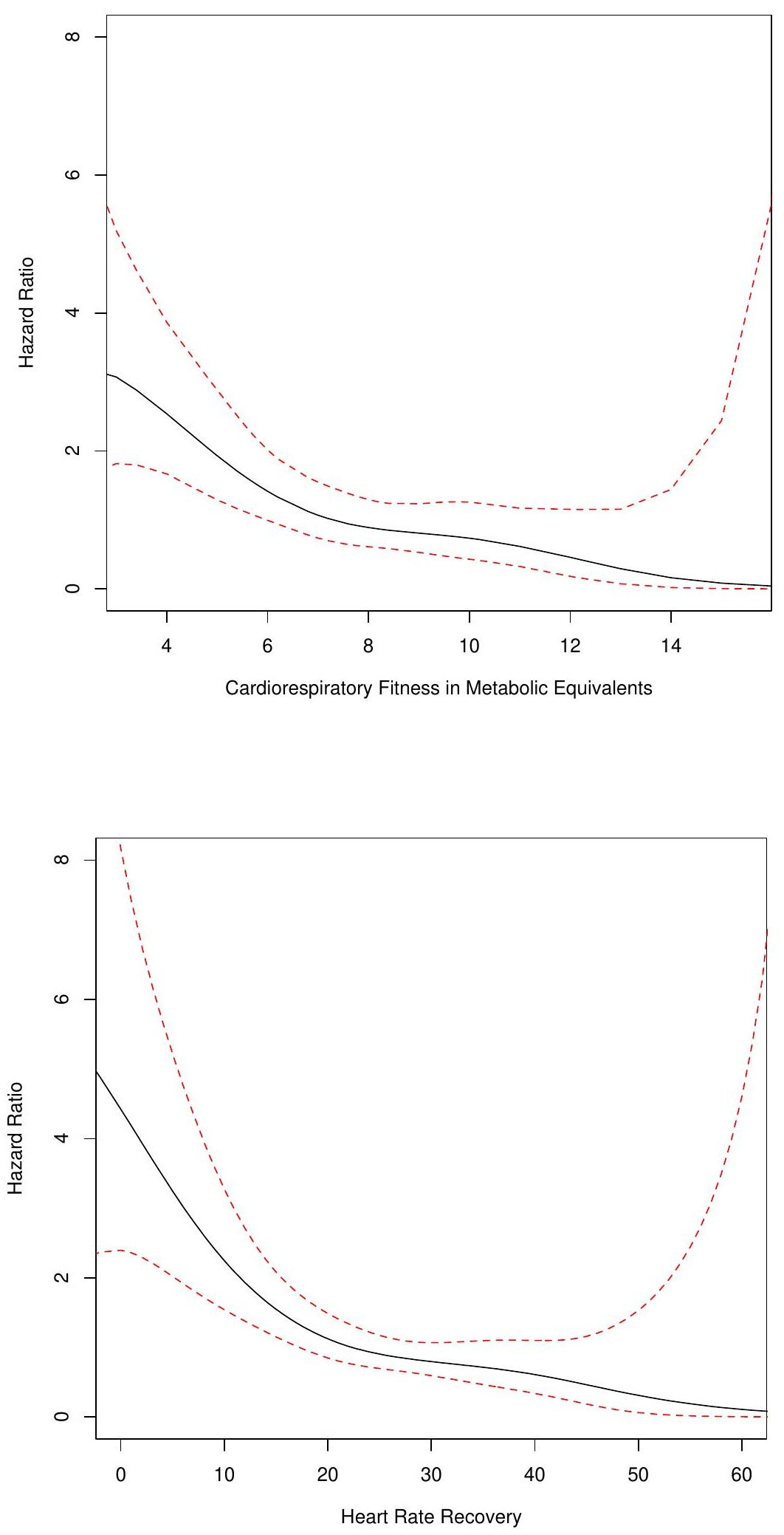